\title{
Capacity and Coverage Tradeoff in WCDMA Environments with Repeaters Deployment
}

\author{
M. GARCIA-LOZANO, L. ALONSO, F. CASADEVALL and S. RUIZ \\ Department of Signal Theory and Communications, Universitat Politècnica de Catalunya (UPC), Barcelona, \\ Spain \\ E-mail: mariogarcia@tsc.upc.edu
}

\begin{abstract}
This work derives the analytic expression of the feasibility condition for the uplink of a WCDMA mobile communications system with repeaters deployment in a multiservice environment with a general heterogeneous layout. In particular, a compact closed expression for the admission region is presented, suitable for a system where the users belong to an arbitrary number of different service classes. A tradeoff between capacity and coverage arises and it has been analysed both theoretically and by means of simulations. Different parameters are shown to have a major impact and their adjustment is discussed.
\end{abstract}

Keywords: radio planning, WCDMA, repeaters, capacity and coverage

\section{Introduction}

Mobile communications systems often work under high heterogeneous conditions, both in time and space domains. This fact makes the proper design of the network infrastructures very important, especially the radio access part. Optimizing this design is a technical and economical challenge. Active repeaters (non-regenerative) are of special interest when considering the deployment of the coverage of a mobile communications system in a certain set of situations that include filling holes in a certain coverage area (including indoor and inside tunnels), or extending the service area beyond its boundaries. Moreover, repeaters are expected to play an important role in planning WCDMA systems because they will also be a cost-effective option to reduce intercell interference. Moreover, the improvement of operation and maintenance capabilities as well as of technologies such as radio over fiber and optical wireless will promote a dense deployment of these devices.

Unfortunately, since a repeater is not a noiseless device, it modifies the interference and thermal noise patterns of the donor base station (BS) receiver (RX). This has and effect on both the coverage of the BS and the capacity. The noise floor of the donor is increased, and consequently its effective cell area is shrunk. Obviously this loss is clearly compensated by the coverage area of the repeaters themselves. However, this noise rise has also an important impact on (W)CDMA systems capacity, as it is shown later.

Although much previous research efforts have been focused on the analysis of CDMAbased systems ([1-3] only to cite a few), not many studies in the literature analyse the effect of repeaters deployment on CDMA mobile communication systems [4-6]. Moreover, these studies consider simplified scenarios with the presence of users that belong to a single service 
class and radio resource management algorithms are usually not considered when evaluating networks performance.

This paper derives the analytic expression of the feasibility condition for the uplink (UL) of a CDMA mobile communications system with repeaters in a multiservice environment with a general heterogeneous layout. In particular, a compact closed expression for the admission region is presented, suitable for a system where the users belong to an arbitrary number of different service classes. This generic analysis could be used for both the deployment of mobile communication systems and the implementation of suitable admission control mechanisms. Moreover, as it is shown later, a tradeoff between capacity and coverage arises and this is analysed both theoretically and by means of Monte Carlo simulations. Different parameters are shown to have a major impact and their adjustment is discussed.

Thus, the organization of the paper is as follows. After this introduction, Section 2 deals with the impact of repeaters on admission control. This is done along two subsections and in a comparative way with environments without repeaters. Section 3 presents simulations and studies the tradeoff between capacity and coverage. Finally, Section 4 closes the paper with the conclusions of the work.

\section{Admission Control in UMTS Systems}

\subsection{Admission Control in Environments without Repeaters}

Admission Control (AC) is a key Radio Resource Management strategy in WCDMA systems. Since coverage and capacity are tightly coupled, a method that handles all new incoming traffic is mandatory. AC strategies decide whether a new radio access bearer (RAB) can be admitted or not according to a certain estimation of the current load. If the load stays below a certain threshold the new RAB will be allowed.

The parameter $\eta(m)$ defined in (1) represents the load factor in the UL measured at BS $m$. It is directly related with the noise rise due to interference levels [7]. Note that the summations are calculated over the MTs connected to BS $m$, this is indicated by ' $i \in m$ ', being $i$ the summation index.

$$
\eta(m)=\frac{\sum_{i=1}^{N_{\mathrm{MT}}} P_{\mathrm{RX}}(m, i)}{\sum_{i=1}^{N_{\mathrm{MT}}} P_{\mathrm{RX}}(m, i)+n_{\mathrm{B}}(m)}=\frac{[1+f(m)] \sum_{\substack{i=1 \\ i=m}}^{N_{\mathrm{MT}}} P_{\mathrm{RX}}(m, i)}{[1+f(m)] \sum_{\substack{i=1 \\ i \in m}}^{N_{\mathrm{MT}}} P_{\mathrm{RX}}(m, i)+n_{\mathrm{B}}(m)}
$$

Where:

- $\quad N_{\mathrm{MT}}$ : Number of mobile terminals (MT) in the system.

- $n_{\mathrm{B}}(m)$ : Thermal noise power at BS $m$.

- $\quad f(m)$ : Quotient between intercell and intracell power, measured at BS $m$.

- $\quad P_{\mathrm{RX}}(m, i)$ : Power received at BS $m$ from MT $i$.

On the other hand, in WCDMA systems, the power control algorithm is composed by the so called inner and outer loops. Whereas the 1st one aims at adjusting the transmitted powers so that a certain signal to interference plus noise ratio $\gamma(k)$ target is reached, the 2 nd one intends to keep the quality of communications at a desired level in terms of block error rate. 
The expression for $\gamma(k)$ measured in the UL before de-spreading is given by (2).

$$
\gamma(k)=\frac{P_{\mathrm{RX}}[s(k), k]}{\{1+f[s(k)]\} \sum_{\substack{i=1 \\ i \in s(k)}}^{N_{\mathrm{MT}}} P_{\mathrm{RX}}[s(k), i]-P_{\mathrm{RX}}[s(k), k]+n_{\mathrm{B}}[s(k)]}
$$

Where:

- $s(k)$ : BS that is serving the MT $k$. Primary station that has a connection with $k$.

On the other hand, and in order to simplify subsequent expressions, we define the parameter $\phi(k)$ as $\gamma(k) /[1+\gamma(k)]$, that is the signal to signal-plus-interference-plus-noise ratio (3).

$$
\phi(k) \equiv \frac{\gamma(k)}{1+\gamma(k)}=\frac{P_{\mathrm{RX}}[s(k), k]}{\{1+f[s(k)]\} \sum_{\substack{i=1 \\ i \in s(k)}}^{N_{\mathrm{MT}}} P_{\mathrm{RX}}[s(k), i]+n_{\mathrm{B}}[s(k)]}
$$

Then, after combining (1) and (3), (4) is obtained, which establishes a relationship between the load factor at BS $m, \eta(m)$, and the summation of all the $\phi$ targets of those users connected with BS $m$.

$$
\eta(m)=[1+f(m)] \sum_{\substack{i=1 \\ i \in m}}^{N_{\mathrm{MT}}} \phi(i)
$$

In order to simplify the expression, we define the ASSIR(m), Aggregated Signal to Signal-plus-Interference-plus-noise ratio:

$$
\operatorname{ASSIR}(m) \equiv \sum_{\substack{i=1 \\ i \in m}}^{N_{\mathrm{MT}}} \phi(i)
$$

And thus, (4) can be simplified and re-written as follows:

$$
\eta(m)=[1+f(m)] \cdot \operatorname{ASSIR}(m)
$$

The expression in (6) has to be always lower than the maximum allowed load factor $\eta_{\max }$, so that interference levels are kept sufficiently low and connections are not degraded. That means the condition in (7) has to be accomplished by all the cells in the system.

$$
\operatorname{ASSIR}(m) \leq \frac{\eta_{\max }}{1+f(m)}
$$

This condition states how many users of each type can be admitted without exceeding the maximum allowed load in the cell, or rather it defines an admission region. Note that the expression is general and independent on the type of service the users are using.

\subsection{Admis sion Control in Environments with Repeaters}

Subsequently, a general deployment with the presence of repeaters in a multiservice scenario is considered. An analytical solution of the feasibility condition for the UL of a CDMA system is derived and compared with that in Subsection 2.1.

As it was previously pointed, repeaters are not noiseless devices, a certain noise rise appears whenever new equipment is installed. Consequently, some changes must be introduced in the 
previous expressions. Particularly, $\phi(k)$ becomes (8).

$$
\phi(k)=\frac{P_{\mathrm{RX}}[s(k), k]}{\{1+f[s(k)]\} \sum_{\substack{i=1 \\ i \in s(k)}}^{N_{\mathrm{MT}}} P_{\mathrm{RX}}[s(k), i]+n_{\mathrm{B}}[s(k)]+n_{\mathrm{R}} \sum_{r=1}^{N_{\mathrm{R}}[s(k)]} G_{\mathrm{g}}[r, s(k)]}
$$

Where:

- $N_{\mathrm{R}}(m)$ : Number of repeaters connected to BS $m$.

- $n_{\mathrm{R}}$ : Thermal noise power at the repeaters.

- $G_{\mathrm{g}}(r, m)$ Global Gain, this is the absolute gain between the repeater and its donor BS (denoted by $m$ ). Note, however, that $s(k)$ appears in (8) because we are not talking about a generic BS $m$, but the one that is serving the MT $k$. This gain considers:

- The internal gain of the repeater itself.

- The gain of the transmitter of the repeater in the link with the BS.

- The gain of the RX of the BS in this link.

- The absolute loss in the link between the BS and the repeater.

Note that, in order to avoid amplifiers saturation at the repeaters, the maximum possible value for $G_{\mathrm{g}}(r, m)$ is the quotient between the repeater maximum transmission power $P_{\mathrm{R}, \max }(r)$ and the BS maximum transmission power $P_{\mathrm{BS}, \max }(m)$. Also note that if $m$ is not the donor BS of the repeater $r$, then $G_{\mathrm{g}}(r, m)=0$.

$$
P_{\mathrm{R}, \max }(r) \geq P_{\mathrm{BS}, \max }(m) \cdot G_{\mathrm{g}}(r, m)
$$

Because of the introduction of an extra noise power, the load factor must be also redefined (10). Moreover, in order to compare with environments without repeaters, the number of users has been maintained to $N_{\mathrm{MT}}$. It is irrelevant if they have established their connection through the donor BS or one of its repeaters, the appropriate value of $P_{\mathrm{RX}}(m, i)$ would be equally adjusted by the power control, but just considering a different effective path loss.

$$
\eta(m)=\frac{[1+f(m)] \sum_{\substack{i=1 \\ i \in m}}^{N_{\mathrm{MT}}} P_{\mathrm{RX}}(m, i)+n_{\mathrm{R}} \sum_{r=1}^{N_{\mathrm{R}}(m)} G_{\mathrm{g}}(r, m)}{[1+f(m)] \sum_{\substack{\mathrm{MT} \\ i \in m}}^{N_{\mathrm{MT}}} P_{\mathrm{RX}}(m, i)+n_{\mathrm{B}}(m)+n_{\mathrm{R}} \sum_{r=1}^{N_{\mathrm{R}}(m)} G_{\mathrm{g}}(r, m)}
$$

In order to allow an easy comparison with (7), a new parameter $\beta(m)$ is defined in (11), which will be introduced in the expressions next.

$$
\beta(m) \equiv \frac{1+\delta F(m) \sum_{r=1}^{N_{\mathrm{R}}(m)} G_{\mathrm{g}}(r, m)}{\delta F(m) \sum_{r=1}^{N_{\mathrm{R}}(m)} G_{\mathrm{g}}(r, m)}
$$

Being $\delta F(m)$ the relationship between the noise figure of the repeaters, $F_{\mathrm{R}}$, and that of the $m$ BS, $F_{\mathrm{B}}(m)$ (typically $F_{\mathrm{B}}(m)<F_{\mathrm{R}}$ ).

$$
\delta F(m) \equiv \frac{F_{\mathrm{R}}}{F_{\mathrm{B}}(m)}=\frac{n_{\mathrm{R}}}{n_{\mathrm{B}}(m)}
$$


As a consequence, the expression in (13) is obtained.

$$
\begin{aligned}
\beta(m) \eta(m)-1 & =\frac{[\beta(m)-1][1+f(m)] \sum_{\substack{i=1 \\
i \in m}}^{N_{\mathrm{R}}(m)} P_{\mathrm{RX}}(m, i)}{[1+f(m)] \sum_{\substack{i=1 \\
i \in m}}^{N_{\mathrm{R}}(m)} P_{\mathrm{RX}}(m, i)+n_{\mathrm{B}}(m)\left[1+\delta F(m) \sum_{r=1}^{N_{\mathrm{R}}(m)} G_{\mathrm{g}}(r, m)\right]} \\
& =[\beta(m)-1][1+f(m)] \operatorname{ASSIR}(m)
\end{aligned}
$$

And thus, a new admission region is found and defined by (14).

$$
\operatorname{ASSIR}(m) \leq \frac{\beta(m) \eta_{\max }-1}{[\beta(m)-1][1+f(m)]}=\frac{\eta_{\max }-\left(1-\eta_{\max }\right)\left[\delta F(m) \sum_{r=1}^{N_{\mathrm{R}}(m)} G_{\mathrm{g}}(r, m)\right]}{1+f(m)}
$$

If this expression is compared with that in the case without repeaters (7), it can be observed that the new admission region is smaller, the system capacity in terms of number of active users is reduced. The new capacity is the one that would be obtained in a network without repeaters but imposing a lower $\eta_{\max }$. This effect is extended and analysed by means of theoretical figures and simulations later on but it is clear that planning a WCDMA network with repeaters will not be such straightforward as in classical 2G FDMA/TDMA networks.

Thus, when considering the presence of repeaters, a new parameter $\xi(m)$, depending on the admission control threshold $\eta_{\max }$, can be defined (15).

$$
\xi(m) \equiv \eta_{\max }-\left(1-\eta_{\max }\right)\left[\delta F(m) \sum_{r=1}^{N_{\mathrm{R}}(m)} G_{\mathrm{g}}(r, m)\right]
$$

And therefore the definition of the admission region can be re-written as follows:

$$
\operatorname{ASSIR}(m) \leq \frac{\xi(m)}{1+f(m)}
$$

Where $\xi(m)$ represents the equivalent $\eta_{\max }$ that should be imposed at the BS $m$ in an environment without repeaters to obtain the same new admission region. It can be seen that the capacity reduction depends on the extra level of noise that is measured at the donor. The higher the number of installed repeaters, their noise figure or the global gain term, the smaller the admission region becomes. Note as well, that this degradation also depends on the maximum allowable load factor, for higher thresholds, the reduction is smaller, unfortunately this is a rather fixed design parameter.

Nevertheless, the conclusions in the previous paragraph are straightforward as long as the relationship between the intra and the intercell power $f(m)$ is maintained after installing repeaters. This is accomplished, for example, by those situations in which repeaters are 'isolated' from the rest of the system, for instance when covering a tunnel or inside a building with high propagation losses. On the other hand, there are other situations in which the installation of these devices modifies $f(m)$. In these cases, the analytical expression of the admission region is identical but substituting $f(m)$ by the new value, $\varphi(m)$. The impact of $\varphi(m)$ on the new admission region should be evaluated for each particular scenario. For example, repeaters could transmit more intercell power to the donor BS and decrease even more the admission region. 
Figure 1 shows some different examples of the facts that have been discussed $(f(m)$ is not modified after installing the repeaters). Indeed, the admission regions are plotted in a particular case of 3 different types of services and values of $\eta_{\max }$. Note that each axis represents one of the first three services in Table 1, depicted later with other simulation conditions. The darker graph represents those possible combinations of types of users that would not exceed a load threshold of 0.8 . On the other hand, the gains are set so that $G_{\mathrm{g}}$ is equal to $0 \mathrm{~dB}$ and the repeaters noise factor is considered to be the same as the donor BS. It can be seen that if one repeater is installed, the number of users would be drastically reduced. Under these assumptions, the number of accepted users would be equal to a situation without repeaters in which the maximum allowable load $\xi$ is 0.6 . If a second repeater is introduced, the equivalent maximum load $\xi$ would be 0.4 . Finally, the installation of four repeaters would imply such a noise rise that no users could be admitted in the cell. The degradation of capacity induced by the installation of repeaters is clear. Moreover, it is interesting that thanks to the definition of $\xi$, there is an easy way to quantify the reduction and compare the network performance before and after installing repeaters.

Figure 2 can also help to the analysis of the plotted admission regions since it shows the maximum allowed load factor and the different equivalent loads $\xi$ that would be obtained for different total received power at the BS. These curves allow an easy comparison of four situations, ranging from 1 to 4 repeaters, all of them with adjusted internal gains so that $G_{\mathrm{g}}$ is $0 \mathrm{~dB}$. It can be seen that the higher the maximum allowed power at the RX, the smaller the differences between $\eta_{\max }$ and the equivalent loads. Also, it can be seen those combinations of $\eta_{\max }$ and number of repeaters which would imply that no users can access the cell, for

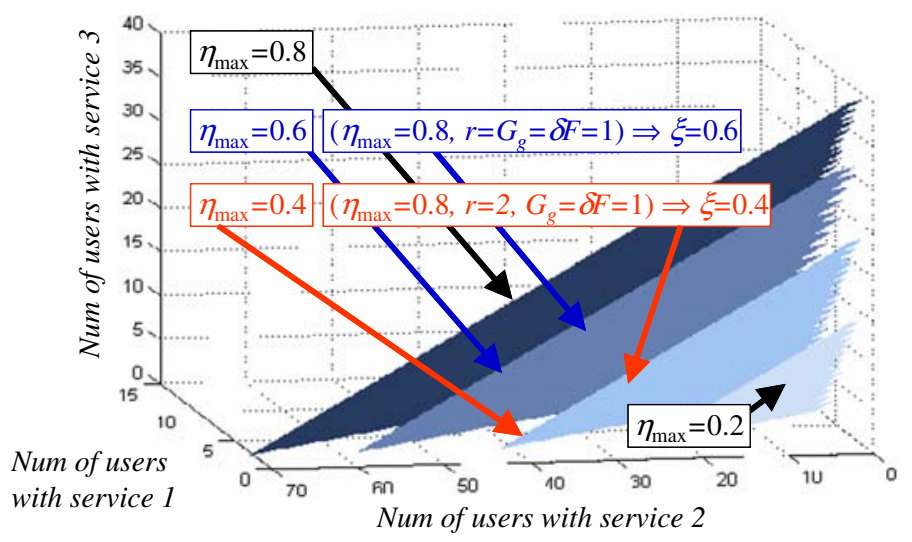

Figure 1. Correspondence of admission regions with and without repeaters.

Table 1. Services features

\begin{tabular}{llll}
\hline Type of service & UL $E_{\mathrm{b}} / N_{0}(\mathrm{~dB})$ & DL $E_{\mathrm{b}} / N_{0}(\mathrm{~dB})$ & Max DL power $(\mathrm{dBm})$ \\
\hline Voice $-12.2 \mathrm{kbps}$ & 2.9 & 4.4 & 21 \\
Voice $-12.2 \mathrm{kbps}(50 \mathrm{~km} / \mathrm{h})$ & 5.5 & 7 & 21 \\
Data $-64 \mathrm{kbps}$ & 1 & 2.5 & 30 \\
Data $-144 \mathrm{kbps}$ & 0.4 & 2.3 & 30 \\
Data $-384 \mathrm{kbps}$ & 0.6 & 2.4 & 30 \\
\hline
\end{tabular}




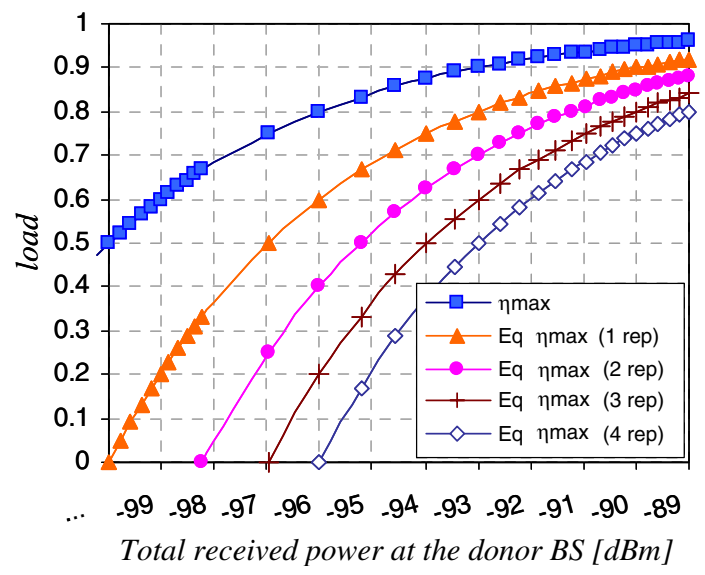

Figure 2. $\eta_{\max }$ and equivalent loads $(\xi)$ as a function of the total received power at the donor BS.

example, when three repeaters are installed and $\eta_{\max }$ must be below 0.75 , or installing four repeaters and imposing $\eta_{\max } \leq 0.8$. Finally, just note that these curves would be scaled by the term $(1+f) /(1+\varphi)$ if the $f$ factor was modified after installing the repeater, see Equation (16).

\section{Capacity and Coverage Tradeoff in WCDMA Systems with Repeaters}

From the previous expressions, it can be stated that:

$$
\lim _{G_{\mathrm{g}} \rightarrow 0} \xi(m)=\eta_{\max } ; \quad \lim _{r \rightarrow 0} \xi(m)=\eta_{\max } ; \quad \lim _{\delta F \rightarrow 0} \xi(m)=\eta_{\max }
$$

This is logical, since all three situations tend to eliminate repeaters noise. However, whereas the third one is imposed by the equipment itself, the first and the second one can be decided during the planning process. Unfortunately, both imply a decrease in coverage so a tradeoff between coverage and capacity arises.

In order to analyse the impact of these parameters on the system performance, static system level simulations have been executed. That is to say, a significant amount of uncorrelated snapshots have been run to obtain statistics. The simulator takes into account both UL and downlink (DL). The scenario is a road-like or railway one in which different numbers of repeaters are added to one BS to cover a certain target area. Classical COST231-Hata propagation model for suburban areas has been used [8] considering a $2 \mathrm{GHz}$ carrier. Regarding the shadowing model, the two dimensional model proposed in Ref. [9] has been introduced with a correlation distance of $18 \mathrm{~m}$ and a standard deviation of $8 \mathrm{~dB}$. Finally, diagram patterns from commercial antennas have been used [10].

Regarding users services, the simulations consider 5 possible types. Table 1 contains their main features. Note that the DL limits the maximum amount of power devoted to one connection. On the other hand, MTs are supposed to have a maximum TX power of $21 \mathrm{dBm}$.

Finally, different realistic types of repeaters have been contemplated, with different maximum TX powers $\left(P_{\mathrm{R}, \max }\right)$. In all cases, their internal gain has been adjusted to the maximum value that guarantees no amplifiers saturation. 


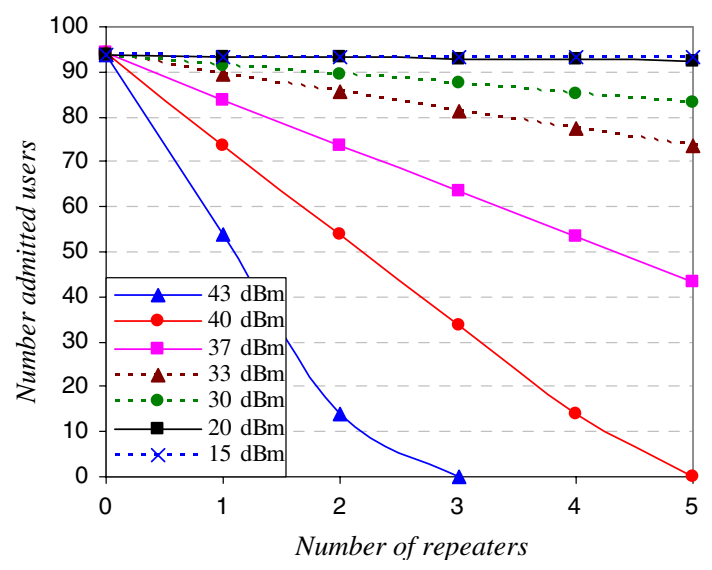

Figure 3. Number of admitter users for different number and types of repeaters.

Figure 3 shows the number of admitted users by the BS as a function of the number of connected repeaters. It can be seen that the reduction in capacity is directly proportional to $P_{\mathrm{R}, \max }$ and the number of repeaters. For example if repeaters transmit with the same maximum power as the BS $(43 \mathrm{dBm})$ the global gain between the repeater and its donor is equal to $0 \mathrm{~dB}$ and therefore repeaters noise is not attenuated at all. This implies a high noise rise at the BS and consequently fewer users can be admitted. In fact, it can be seen that, for certain configurations and number of repeaters, no users could access the system. On the other hand, very low power repeaters do not degrade capacity as long as their number is not over 5, so the impact of the global gain is much more important than the number of installed repeaters. This fact will be analysed again later.

The curves in Figure 4 show coverage in terms of $E_{\mathrm{c}} / I_{0}$, measured on the Common Pilot Channel (CPICH). In our simulations, the pilot channel is realistically introduced according to UMTS networks. The CPICH allows the MT to execute the cell selection and soft handover procedures [11] and therefore a correct reception is mandatory in the target area of coverage. If the $E_{\mathrm{c}} / I_{0}$ measured on the pilot channel at one pixel is over $-12 \mathrm{~dB}$ with a probability higher than $95 \%$, then that position is considered to be correctly covered in terms of CPICH. The figure shows a clear tradeoff, since those situations with higher capacity degradation are the ones with better pilot reception. Nevertheless, low power repeaters allow clear increases of coverage and with those configurations there was no significant loss in capacity. Thus, it seems that the tradeoff can be mitigated by means of many low power repeaters. However, this fact might obviously jeopardize a third parameter to optimize: the network cost.

Although the $E_{\mathrm{c}} / I_{0}$ level is usually admitted as a metric of networks coverage, both UL and DL are limited in power. So CPICH coverage does not guarantee service availability. In fact, Figure 5 shows the medium power that a static voice user has to transmit as a function of its distance to the BS. The black horizontal line shows the maximum available UL TX power. It can be seen that users would have to commute to a degraded mode from around $3500 \mathrm{~m}$ if no repeaters are installed in the scenario. Three situations with four repeaters are shown too. It can be seen that users would have enough power until around $11 \mathrm{~km}$ with $37 \mathrm{dBm}$ repeaters. With $30 \mathrm{dBm}$ repeaters, some deadspots would rise in the line between the donor and the last repeater. Finally, with $15 \mathrm{dBm}$ repeaters, the extra coverage would be very low. Keeping these facts in mind, Figure 6 shows the probability that a service is available (that is, $\mathrm{CPICH}$ 


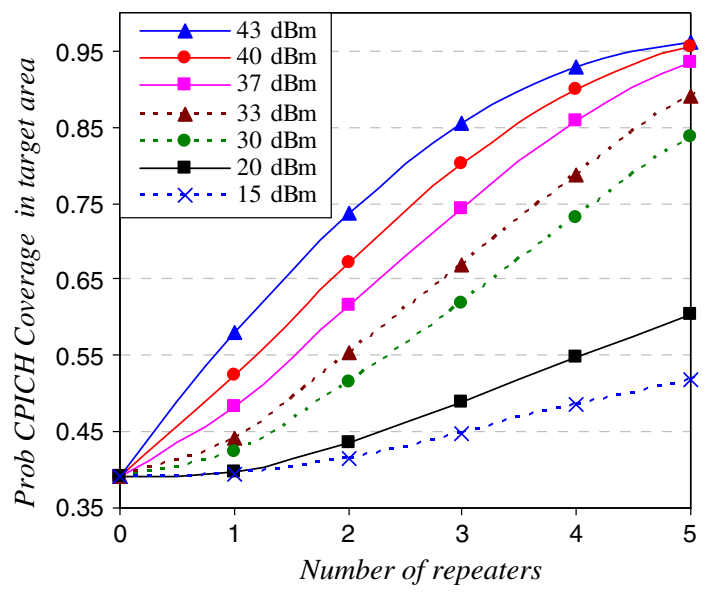

Figure 4. Probability of CPICH coverage in target area.

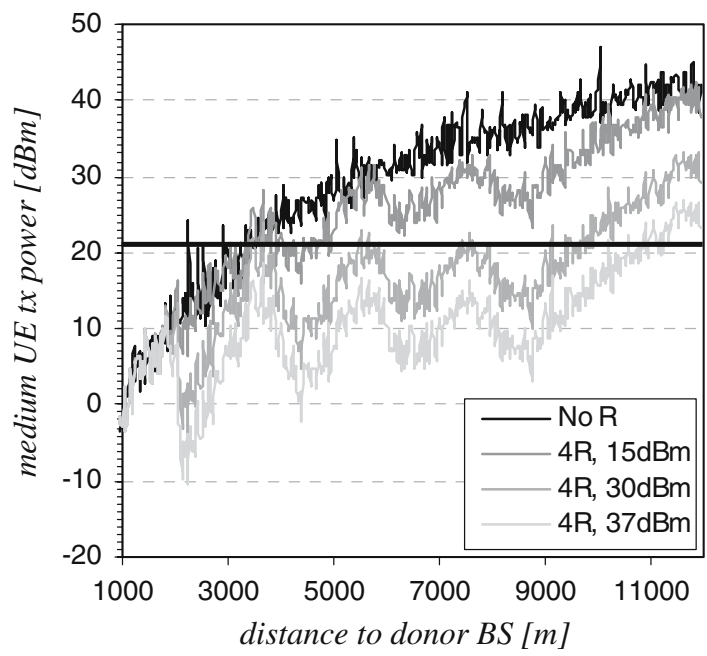

Figure 5. Medium UL transmitted power as a function of the distance to the donor BS.

coverage is satisfactory and the power control does not require more than the maximum power in the UL and in the DL). The figure represents the values for repeaters with 37 and $15 \mathrm{dBm}$ of $P_{\mathrm{R}, \max }$. The notation $S i$ stands for service $i$, where $i$ is the row in Table 1 . It can be seen that the probability increases monotically with $P_{\mathrm{R}, \max }$ and the number of repeaters. However, as expected, both absolute values and increases are quite lower in the $15 \mathrm{dBm}$ case, which shows modest improvements in the services availability.

The advantage of using low power repeaters can be seen in Figure 7. This shows those configurations (number of repeaters and $P_{\mathrm{R}, \max }$ ) that would succeed in covering a certain target area (65\% of the scenario) along with the reduction that they would imply in terms of capacity. For example, if a $10 \%$ reduction is tolerated, 4 repeaters with $P_{\mathrm{R}, \max }=30 \mathrm{dBm}$ could be planned. But if degradation is desired to be below $2.5 \%$, this configuration would not be valid whereas six repeaters with $P_{\mathrm{R}, \max }=20 \mathrm{dBm}$ would accomplish the requisite. The 


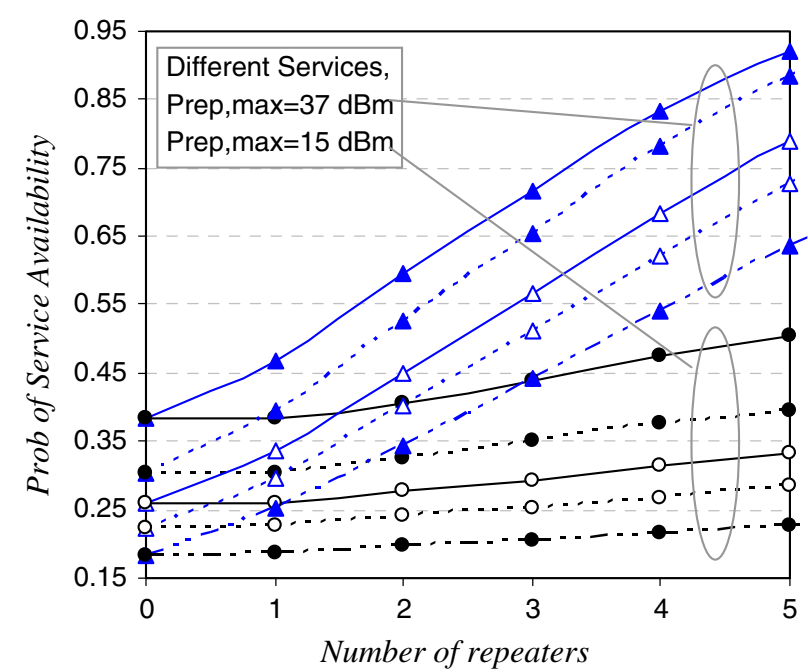

\begin{tabular}{|c|c|}
\hline $\begin{array}{r}-\mathrm{S} 1,37 \mathrm{dBm} \\
\ldots \\
\ldots \\
\ldots\end{array}$ & 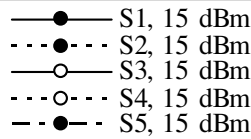 \\
\hline
\end{tabular}

Figure 6. Probability of service availability.

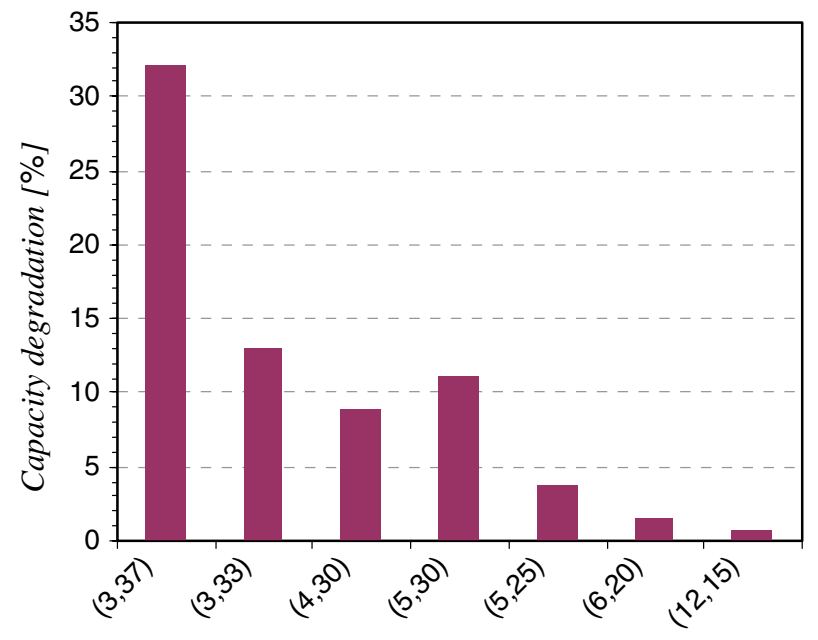

Configurations (num of repeaters, Prep,max)

Figure 7. Degradation of capacity when guaranteeing coverage in a $65 \%$ of the scenario, for different number of repeaters and $P_{\mathrm{R}, \max }$.

desired coverage could be guaranteed without a noticeable capacity loss but, as pointed out, with a much more costly solution. Indeed, 12 low power repeaters $\left(P_{\mathrm{R}, \max }=15 \mathrm{dBm}\right)$ would be a valid configuration because of the high attenuation suffered by the noise. 


\section{Conclusions}

Along this work an analytic expression of the feasibility condition for the UL of a WCDMA mobile communications system with repeaters has been obtained. A compact closed and generalist expression for the admission region has been presented and a new parameter $\xi$ has been defined in order to quantify the resulting capacity degradation. It has been pointed out that planning WCDMA networks with repeaters implies a tradeoff between capacity and coverage, that is why introducing these devices will not be such straight forward as in classical FDMA/TDMA 2G systems. Repeaters noise figure, the global gain in the link between each repeater and the donor, and the number of repeaters itself are parameters with high impact on this tradeoff. The global gain shows an outstanding influence on capacity and service availability, however, by means of simulation it has been seen that degradation is not such significant with low power repeaters. In these situations, a high noise rise can be avoided and the tradeoff is partially controlled. Installation of many repeaters could guarantee both coverage and capacity as long as their internal gains are adjusted so that the global gain is low. The cost of the network, however, might invalidate some of the solutions and certain capacity or coverage reduction could have to be accepted.

\section{Acknowledgments}

This work has been funded by the Spanish Research Council CICYT + FEDER through the projects TEC2005-07326-C02-01/TCM and TIC2003-08609. Part of this work was originally presented in the Wireless Personal Multimedia Communications conference 2005.

\section{References}

1. J. Zander, "Performance of Optimum Transmitter Power Control in Cellular Radio Systems", IEEE Transactions on Vehicular Technology, Vol. 41, no. 1, pp. 57-62, Febuary 1992.

2. S. A. Jafar and A. Goldsmith, "Adaptive Multirate CDMA for Uplink Throughput Maximization", IEEE Transactions on Wireless Communication, Vol. 2, no. 2, pp. 218-228, March 2003.

3. A. Baier, U. C. Fiebig, W. Granzow, W. Koch, P. Teder, and J. Thielecke, "Design Study for a CDMAbased Third-generation Mobile Radio System", IEEE Journal on Selected Areas in Communication, Vol. 12, pp. 733 -743, May 1994.

4. W. Lee and D. Lee, "The Impact of Repeaters on CDMA System Performance", in Proc. of IEEE Vehicular Technology Conf., 2000 (VTC 2000), Tokyo (Japan), pp. 15-18, May 2000.

5. M. Rahman and P. Ernstrom, "Repeaters for Hotspot Capacity in DS-CDMA Networks", IEEE Transactions on Vehicular Technology, Vol. 53, no. 3, pp. 626-633, May 2004.

6. T. W. Ban, B. Y. Cho, W. Choi, and H. Cho, "On the Capacity of a DS-CDMA System with Automatic On-Off Switching Repeaters”, in Proc. of IEEE International Conf. on Comm., 2001 (ICC 2001), Helsinki (Finland), pp. 11-15, June 2001.

7. H. Holma and A. Toskala, WCDMA for UMTS Radio Access for Third Generation Mobile Communications 2nd ed., Ed. Wiley \& Sons, 2002.

8. P. E. Mogensen, P. Eggers, C. Jensen, and J. B. Andersen, "Urban Area Radio Propagation Measurements at 955 and $1845 \mathrm{MHz}$ for Small and Microcells", in Proc. of IEEE Global Telecomm. Conf., 1991, (GLOBECOM 1991) Phoenix (USA), pp. 2-5, December 1991.

9. R. Fraile, O. Lázaro, and N. Cardona, “Two Dimensional Shadowing Model”, COST-273, Report TD(03)-171, Prague, Czech Rep., pp. 24-26, September 2003 (http://www.lx.it.pt/cost273/).

10. http://www.kathrein.de/ 
11. 3GPP Specification. TS 25.133 (Release5) - "Requirement for Support of Radio Resource Management (FDD)" http://www.3gpp.org/

12. 3GPP Specification. TR 25.956 (Release4) - "UTRA Repeaters Planning Guidelines and System Analysis" http://www.3gpp.org/

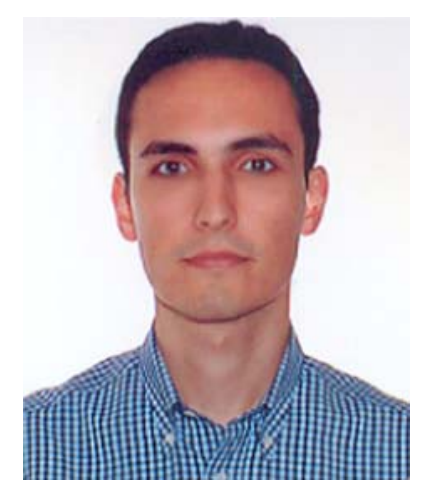

Mario Garcia-Lozano received the Degree in Telecommunications Engineering from the Technical University of Catalonia (UPC), Spain, in 2001. Currently, he is working toward the Ph.D. degree in Telecommunications Engineering. From 1999 to 2002 he was a member of the technical staff at Retevision, Spain where he worked on the design and development of LMDS networks. In 2002, he joined the Department of Signal Theory and Communications at UPC, where he worked as a Research Assistant until 2003 under a grant from the Catalan Government. He currently lectures at the Castelldefels School of Technology (UPC). He has actively participated in several research projects, funded by the European Union, the Spanish and Catalan Governments and private companies. His research activities are focused on the field of mobile communication systems, especially radio network planning and radio resource management issues and optimization of cellular networks.

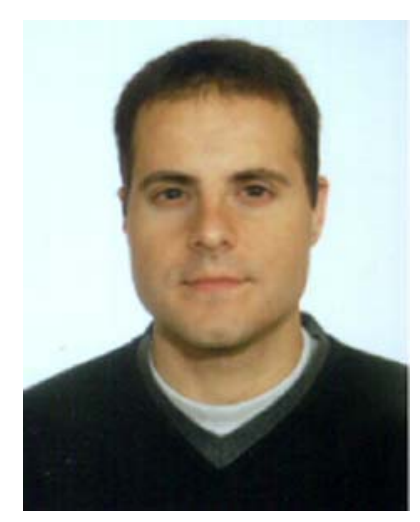

Luis Alonso received his Ph.D. in telecommunications engineering from the Technical University of Catalonia (UPC), Spain, in 2001, where he is Associate Professor in the Radio Communications Group of the Signal Theory and Communications Department. He participates in research programs and projects funded by the European Union and the Spanish Government, and also collaborates with some telecommunications companies. His current research interests are in the field of medium access protocols, radio resource management, cross-layer optimization and QoS features for all kind of wireless communications systems. 


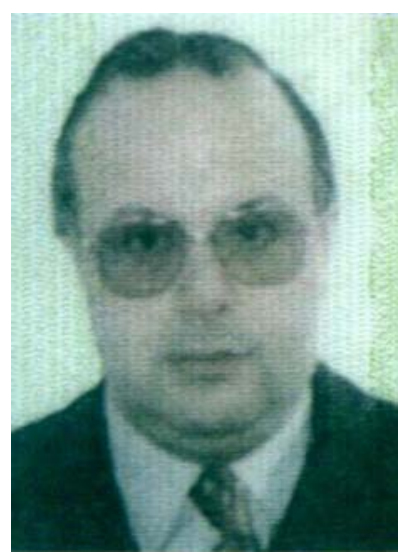

Fernando J. Casadevall received the Engineer of Telecommunication and Dr. Engineering degrees from the Universitat Politècnica de Catalunya (UPC), Spain, in 1977 and 1983, respectively. In 1978 he joined UPC, where he was an Associate Professor from 1983 to 1991. He is currently Full Professor in the Signal Theory and Communications Department.

After graduation he was concerned with equalization techniques for digital fibre optic systems. He has also been working in the field of digital communications with particular emphasis on digital radio and its performance under multipath propagation conditions. In the last 15 years, he has mainly been concerned with the performance analysis and development of digital mobile radio systems. In particular his research interest include cellular and personal communication system, multipath transceiver design (including Software Radio techniques), Mobility and Radio Resources Management, End to end QoS issues.

During the last 10 years he participated in more than 30 research-projects funded by both public and private organizations. In particular, he actively participated in 10 research projects founded by the European Commission, being the Project Manager of three of them: ARROWS, EVEREST and AROMA (see http://www.gcr.tsc.upc.edu for details).

Prof. Casadevall has published around one hundred of technical papers in both International Conferences and Magazines; most of them correspond to IEEE publications. He has also been Technical Program Committee member of different international IEEE supported conferences as well as reviewer of several IEEE magazines.

From October 1992 to January 1996, he was responsible for the Information Technology Area in the National Agency for Evaluation and Forecasting (Spanish Nation Research Council). 


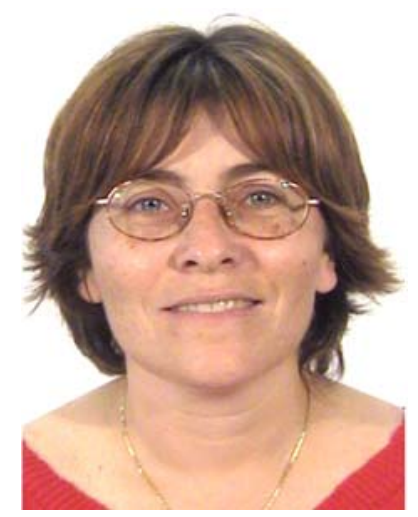

Silvia Ruiz received the Engineer and Doctor Engineer degrees in Telecommunication from the Technical University of Catalonia (UPC), Spain, in 1986 and 1989, respectively. He joined the Signal Theory and Communications department becoming Associate Professor in 1992. She has participated in several UE projects, COST actions and Network of Excellence, as well as in projects funded by the Spanish government and private projects (Alcatel, Vodafone. Telefónica Móviles, etc.). She has also strongly collaborated in university management issues and has been Vice-Dean of Academic Affairs for 3 years, and Assistant Director of External Relations of the Castelldefels School of Technology since December 2005 (UPC). Her research interests are in the field of mobile communication systems, especially radio network planning issues and optimization of $3 \mathrm{G}$ and heterogeneous networks. 